\title{
Paper-Supported High-Throughput 3D Culturing, Trapping, and Monitoring of Caenorhabditis Elegans
}

\author{
Mehdi Tahernia $®$, Maedeh Mohammadifar and Seokheun Choi * \\ Bioelectronics \& Microsystems Laboratory, Department of Electrical \& Computer Engineering, State University \\ of New York-Binghamton, Binghamton, NY 13902, USA; mtahern1@binghamton.edu (M.T.); \\ mmoham11@binghamton.edu (M.M.) \\ * Correspondence: sechoi@binghamton.edu
}

Received: 2 January 2020; Accepted: 15 January 2020; Published: 17 January 2020

\begin{abstract}
We developed an innovative paper-based platform for high-throughput culturing, trapping, and monitoring of $C$. elegans. A 96-well array was readily fabricated by placing a nutrient-replenished paper substrate on a micromachined 96-well plastic frame, providing high-throughput 3D culturing environments and in situ analysis of the worms. The paper allows $C$. elegans to pass through the porous and aquatic paper matrix until the worms grow and reach the next developmental stages with the increased body size comparable to the paper pores. When the diameter of C. elegans becomes larger than the pore size of the paper substrate, the worms are trapped and immobilized for further high-throughput imaging and analysis. This work will offer a simple yet powerful technique for high-throughput sorting and monitoring of $C$. elegans at a different larval stage by controlling and choosing different pore sizes of paper. Furthermore, we developed another type of 3D culturing system by using paper-like transparent polycarbonate substrates for higher resolution imaging. The device used the multi-laminate structure of the polycarbonate layers as a scaffold to mimic the worm's 3D natural habitats. Since the substrate is thin, mechanically strong, and largely porous, the layered structure allowed C. elegans to move and behave freely in 3D and promoted the efficient growth of both C. elegans and their primary food, E. coli. The transparency of the structure facilitated visualization of the worms under a microscope. Development, fertility, and dynamic behavior of C. elegans in the 3D culture platform outperformed those of the standard 2D cultivation technique.
\end{abstract}

Keywords: C. elegans; paper-based platforms; transparent polycarbonate substrate; 3D culturing environments; high-throughput

\section{Introduction}

Growing small organisms such as human cells and bacteria under carefully controlled conditions, generally outside their natural habitats, is a key step for tissue engineering, drug screening and development, and disease modeling [1]. Cultures have been mainly carried out on 2D platforms, such as petri dishes, microtiter plates, and flasks, in which the organisms are placed onto a flat surface that is different from in vivo 3D environment [2]. Although the conventional 2D culture techniques generated a wealth of new scientific and technological results with significant and transformative potential, they fail to provide an accurate structure, function, or physiology of living organisms and even worse can alter their gene expression, metabolism, production, and morphology compared to those in 3D [1-4]. These organisms inherently respond to a variety of chemical and physical cues from their 3D microenvironment differently than 2D cues while performing the fundamental cellular processes such as proliferation, differentiation, apoptosis, and migration. Therefore, mimicking the 3D environment is required to stimulate the actual in vivo growth conditions, provide an accurate model of the living organisms, and thus revolutionize bioengineering and biomedical applications. 
Among many small organisms, Caenorhabditis elegans is widely used as a model to study mechanisms of neurobiological processes and human diseases $[5,6]$. As many of the cellular and molecular processes in C. elegans have proven relevant for human biology, the worm has been an important model system to study the mechanisms of disease and perform drug discovery experiments. However, C. elegans have been notoriously unable to be manipulated in the 3D microenvironment. There are technical challenges to control nutrient and gas transport in 3D and provide a 3D scaffold for C. elegans having different sizes in terms of their developmental stages (egg, L1, L2, L3, L4, and adult stages). Developmental transitions in C. elegans and their reproduction efficiencies rely extensively on culturing conditions. Typically, C. elegans is cultured using the E. coli strain OP50 as a food source on a standard 2D Nematode growth medium (NGM) agar [7]. However, the worms cultured on a 2D plate are not accurate models of those in their in vivo environments such as rotting fruits and vegetation, and there are limitations in understanding their 3D locomotion behavior at different life stages during cultivation. Therefore, there is a need to develop 3D culture methods for $C$. elegans. High-throughput 3D cultivation of $C$. elegans can be a more powerful technique to identify various genes or drugs potentially relevant to human biology or diseases [8]. Additionally, their mechanical immobilization in their physiologically active state is beneficial for high-resolution microscopic monitoring $[9,10]$. However, reported work on the 3D culture technique with trapping and monitoring of C. elegans in a high-throughput manner is quite limited. High-throughput culturing tools and trapping methods for C. elegans have historically developed separately, and the techniques developed for those applications are usually incompatible with each other [11,12]. Even the latest advances in a 3D controlled environment do not accurately replicate their natural habitats and do not show any significant difference in culturing compared to the standard 2D method [3].

Here, we created a new 3D, high-throughput technique to accomplish all culturing, trapping, and monitoring functions for C. elegans by using paper as a 3D scaffold. A 96-well array was readily fabricated by placing a nutrient-replenished paper substrate on a micromachined 96-well plastic frame, providing high-throughput 3D culturing environments and in situ analysis of the worms. Paper allows C. elegans to pass through a porous and aquatic matrix until the worms grow and reach the next developmental stages with the increased body size comparable to the paper pores. When the diameter of $C$. elegans becomes larger than the pore size of the paper substrate, the worms are trapped and immobilized for further high-throughput imaging and analysis. To facilitate visualization of the works, we also constructed another type of culture system by using paper-like transparent polycarbonate substrates. The system used a multi-laminate structure of thin polycarbonate layers as a scaffold to mimic the worm's 3D natural habitat. We observed that development, fertility, and dynamic behavior of $C$. elegans in the 3D culture system outperformed those of the standard 2D cultivation technique.

\section{Results and Discussion}

The integrated technology for cultivation, immobilization, and monitoring of C. elegans will help promote barrier-transcending and translational advances in studying them. Recently, paper has been used as a substrate for 3D cell culturing and high-throughput biological and chemical analysis because of its intrinsic advantages including low cost, disposability, and easy fabrication [13-15]. Moreover, paper is attractive because it wicks fluids without external pumps and tubes [15]. In addition, paper's well-defined pore sizes can filter to separate different-sized molecules from samples [16,17]. Despite its vast potential, the promise of paper has never been used for $C$. elegans studies.

\subsection{High-Throughput Culturing, Trapping, and Monitoring of C. elegans}

In this work, we demonstrated a 3D, high-throughput paper-based platform to realize all culturing, trapping, and monitoring functions for C. elegans. Spatially-distinct 96 wells of an array were prepared by sandwiching a filter paper substrate for $C$. elegans' habitats and micro-machined polymethyl methacrylate (PMMA) supporting frames for high-throughput chambers (Figure 1). After the paper was filled with Luria-Broth (LB) media, the E. coli strain OP50 was introduced as a food source for the 
worms. We then transferred C. elegans at the first larval stage (L1) to the paper and cultivated them (Figure 2a). Once their body size reached the pore size of the paper, the C. elegans were trapped with low movement capability (Figure 2b,c).

(a)

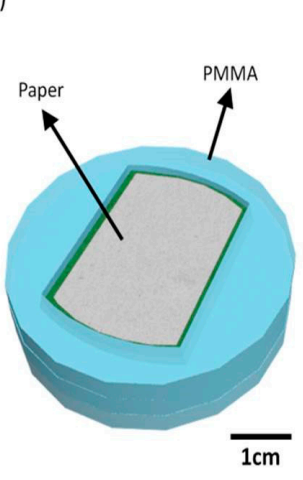

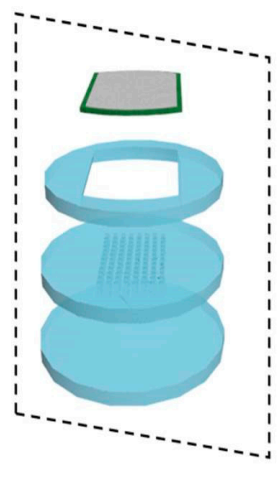

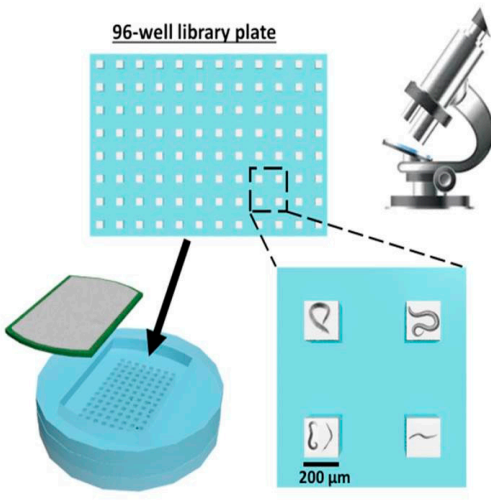

(b)

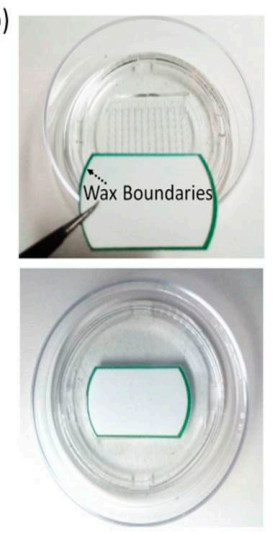

Figure 1. (a) Schematic diagrams and (b) photos of the proposed paper-based culture platform for C. elegans studies.

We used C. elegans with the reporter genes green fluorescent protein (GFP) for visualization. The transparency of $C$. elegans allowed for microscopic analysis in vivo. After $24 \mathrm{~h}$ cultivation, a sufficient number of the worms were reproduced and spread over the paper substrate. Each well contained at least one worm for further experiments (Figures 2a and $3 a, b)$. The porous structure of paper allowed C. elegans to move and behave freely in 3D and promoted the efficient growth of C. elegans and their primary food, E. coli bacteria. When C. elegans becomes larger than the pore size of the paper $(\sim 10 \mu \mathrm{m})$, the worms are trapped and immobilized (Figure $2 \mathrm{~b})$. As the worm body size is typically used as a physiological marker to identify size-related phenotype [18], controlling and choosing different pore sizes of paper can be a powerful technique to analyze the potential biophysical and biochemical mechanisms of $C$. elegans at a different larval stage.

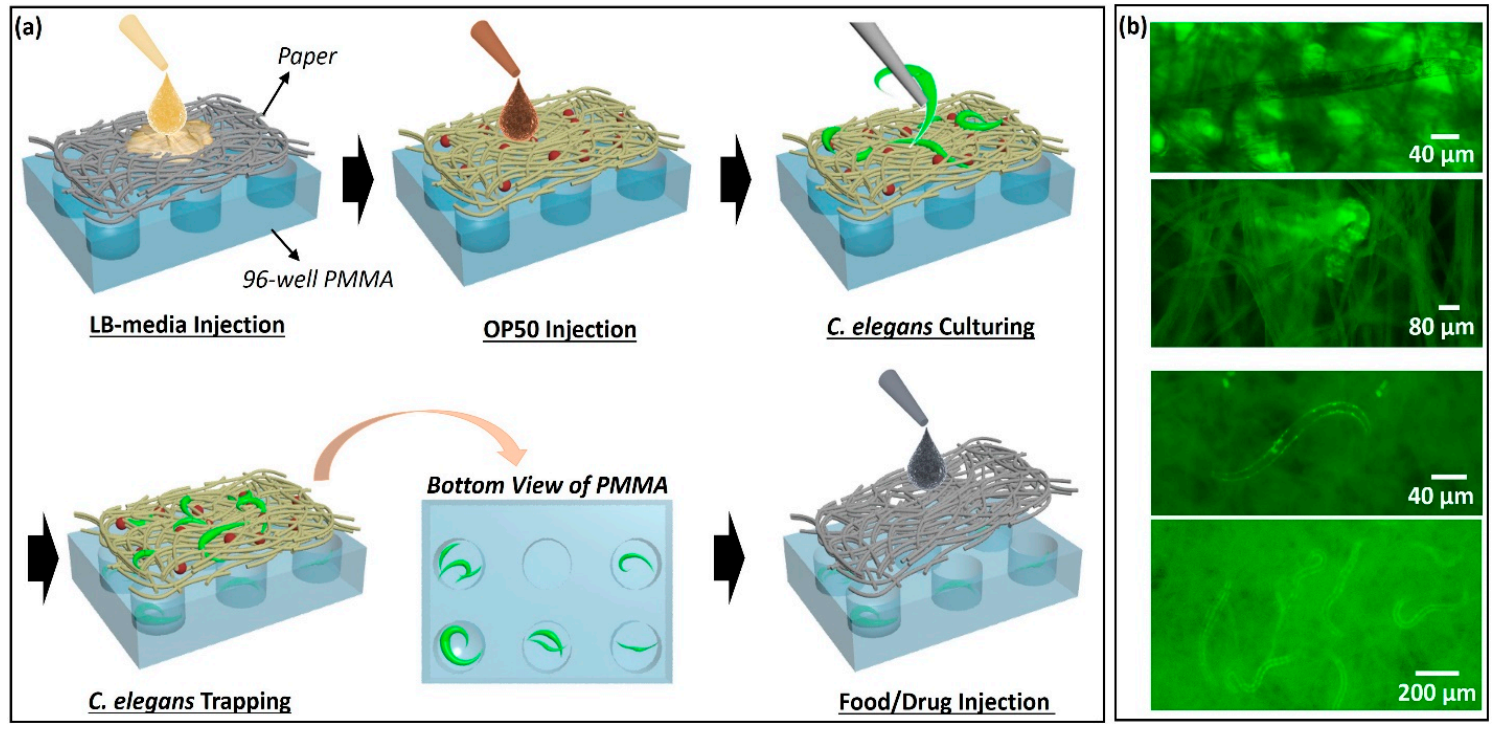

Figure 2. (a) Fabrication procedures, (b) immobilized C. elegans in paper substrates. 

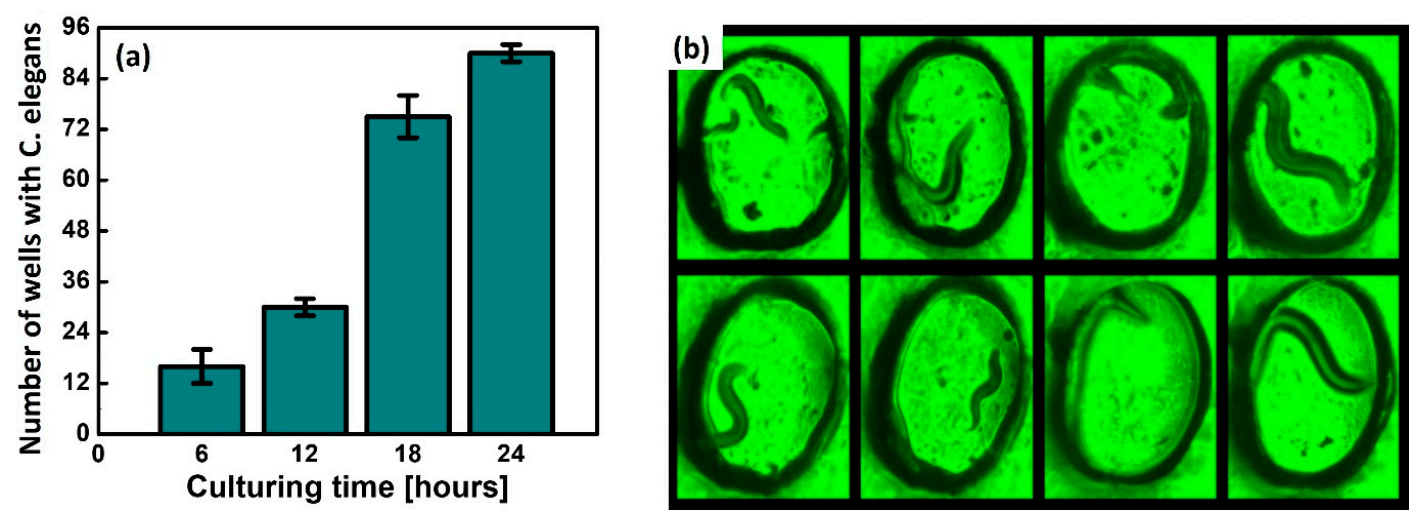

Figure 3. (a) Number of wells filled with cultured worms and (b) microscopic images of trapped C. elegans.

\subsection{Transparent 3D Culture Systems for C. elegans}

Although we visualized the trapped C. elegans by using the GFP (Figure 2b), the opacity of paper did not let much light pass through and did not provide high-resolution images in 3D. To enable direct and visual observation, we used paper-like transparent polycarbonate substrates for the culture system [19]. The system used the multi-laminate structure of the substrates to mimic the worm's in vivo 3D environment. Since the substrate is thin, mechanically strong, and largely porous, the system is the same as our previous one with paper (in Section 2.1) but is transparent to facilitate visualization of the worms under a microscope (Figure S1). The system was constructed by stacking five polycarbonate layers patterned with cuts. Each layer had differently patterned cuts that were perpendicularly crossed over forming small square-shaped through-holes $(\sim 500 \times 500 \mu \mathrm{m})$ at the intersections (Figure 4a). The worms could move vertically through the stack and horizontally along the grids during cultivation. The thick stack provided a 3D aquatic environment holding sufficient amount of LB media for the worms and E. coli. The stack was designed to have nine culturing wells $(\sim 1 \mathrm{~cm}$ diameter) with two PMMS supporting frames (Figure 4). The capillary wicking properties of porous polycarbonate substrates enabled controlled and uniform mass transport of nutrients and gasses for C. elegans while their optical transparency allowed real-time monitoring under the microscope without the GFP expression (Figure $4 b, c$ ). Moreover, this thin, cost-effective, and disposable polycarbonate substrate permits easy cleaning, sterilizing, and microfluidic pathways. Finally, the stack can be assembled in modular fashion, which allows us to control the thickness of the overall construct and diffusion of nutrients through the stack. Reversely, the simple de-stacking can promote easy C. elegans sampling for further experiments. 


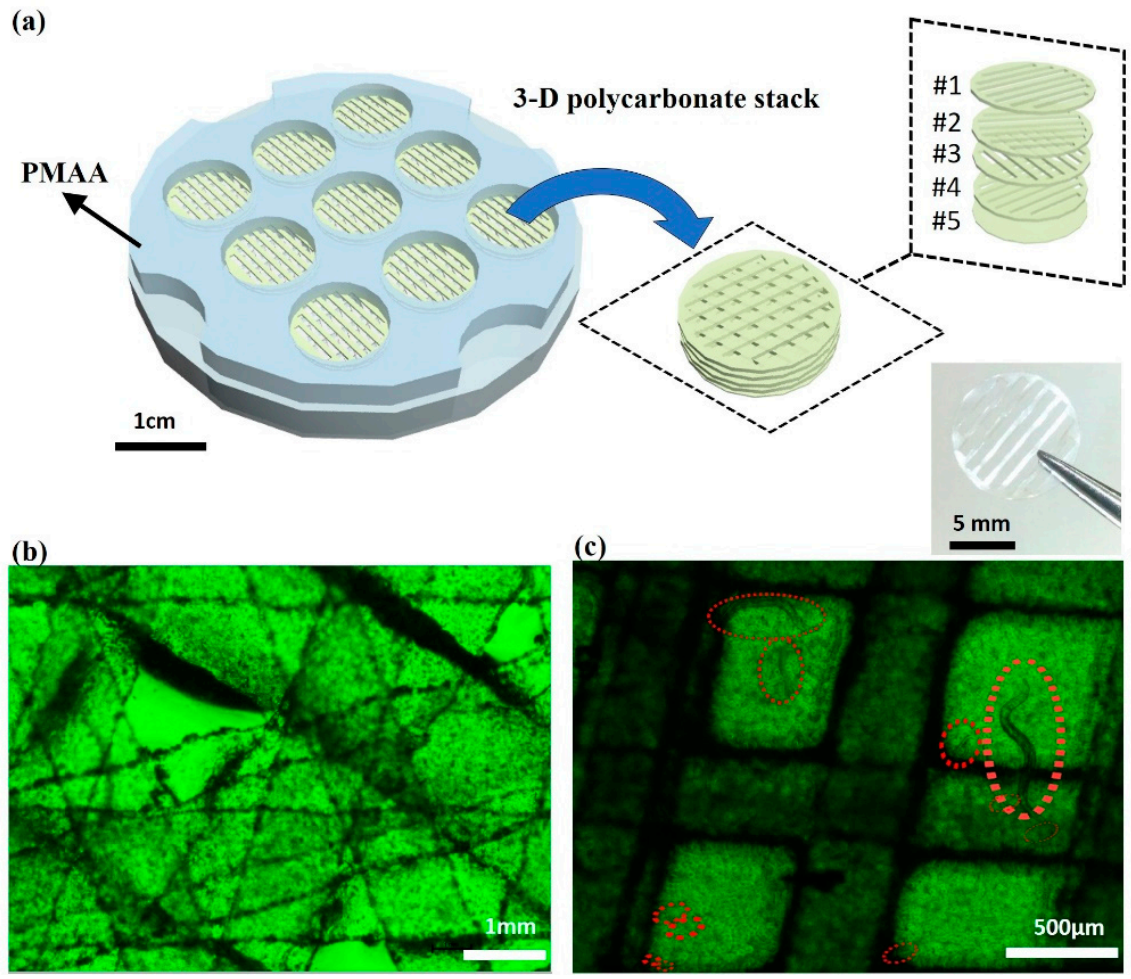

Figure 4. (a) Schematic diagrams of the 3-D culture system for C. elegans and microscopic images of the culture regions (b) without and (c) with C. elegans.

\subsection{D Behavioral Dynamics of C. elegans}

To demonstrate the performance of the 3D culture system, we prepared three different culturing systems (i) conventional 2D agar plate, (ii) 3D polycarbonate stack, and (iii) 3D natural habitat (i.e., grape) and compared C. elegans' activity and average speed (Figure 5) in each culturing system. The $C$. elegans' movements were recorded to measure their average speed and the area that the worm crawls about. The grape provided a transparent culturing environment enough to efficiently analyze the worms' movements. In particular, the worms demonstrated vertical bending activities and movements in 3D platforms while their predominant activity is a horizontal bending on the 2D agar plate. Figure 5 shows the normalized average areas and speeds of $C$. elegans in three environmental systems for five days of culturing, which were measured by WormLabß3.0 software (MBF Bioscience, Williston, VT, USA). The worms on the 2D agar plate showed much higher speed $(\geq 350 \mu \mathrm{m} / \mathrm{s})$ with a smaller moving area because of their limited horizontal bending activity ( $\leq 2$ body area/s) than other 3D systems. The area that the worms moved per second in the grape and in the polycarbonate stack was $\sim 4.2$ and $\sim 3.0$, respectively, which is much higher than that of the 2D agar plate. Our 3D polycarbonate culture system showed very similar moving activities and speed to the in vivo fruit environment. Interestingly, the worms' movement speed on the 2D platform was lower at the beginning of culture (Day 1) compared to those on following days after creation of paths throughout the plate while the other 3D systems have similar speed through the culturing days. This indicates that the 2D platform cannot simulate the actual in vivo environment. 
(a) 2-D agar plate

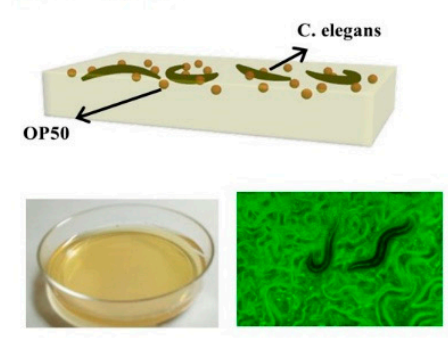

(b) 3-D polycarbonate stack

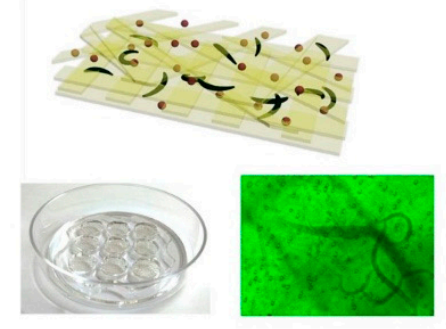

(c) 3-D natural habitat (grape)

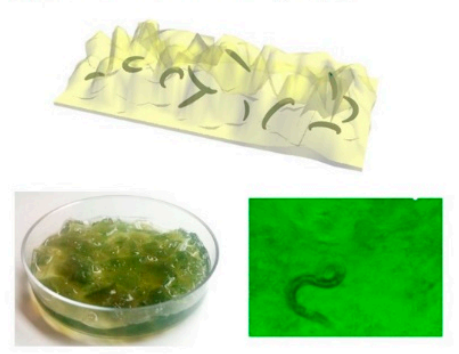

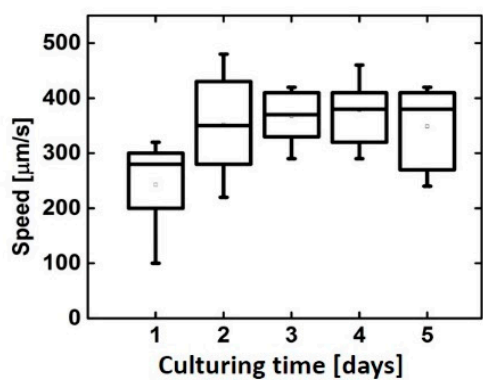
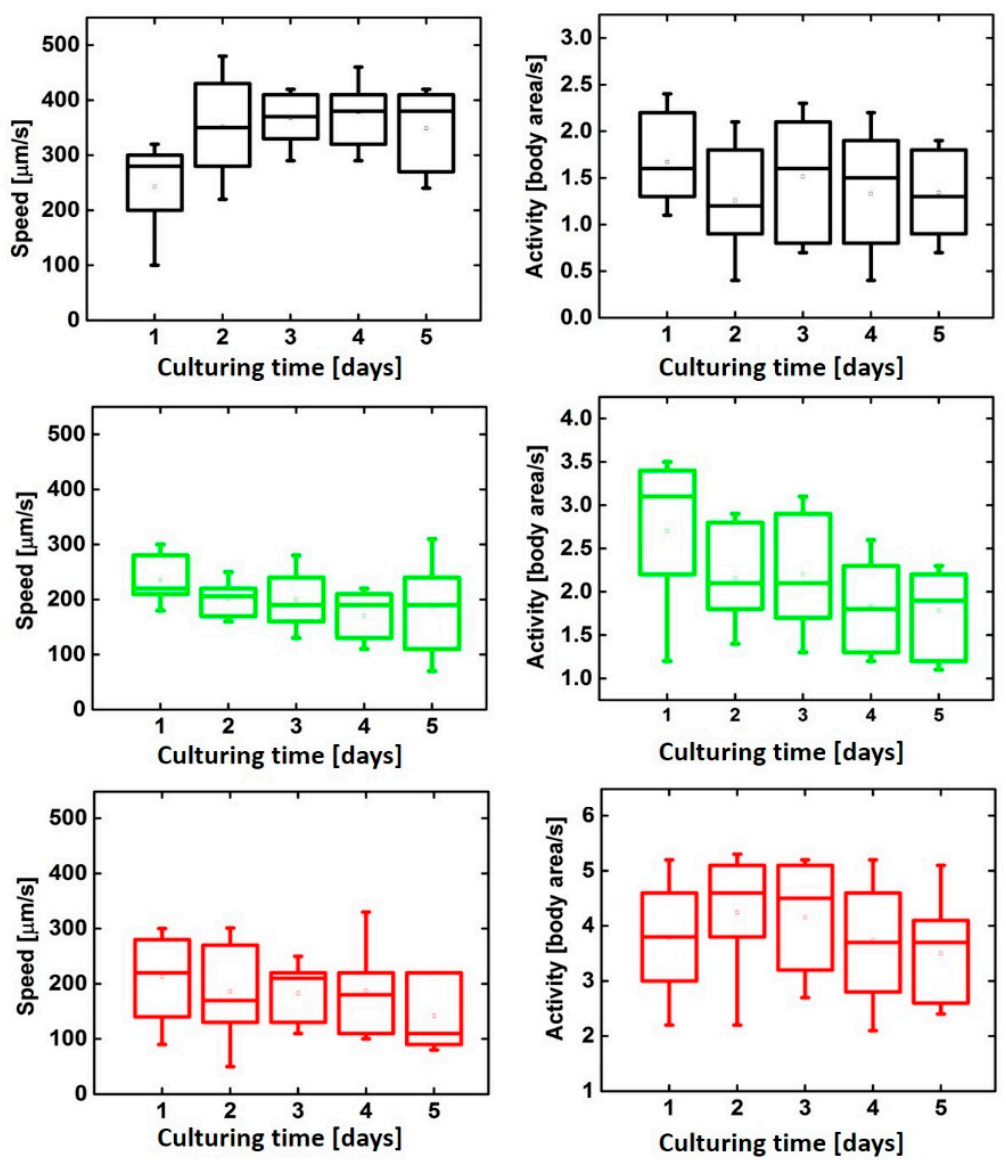
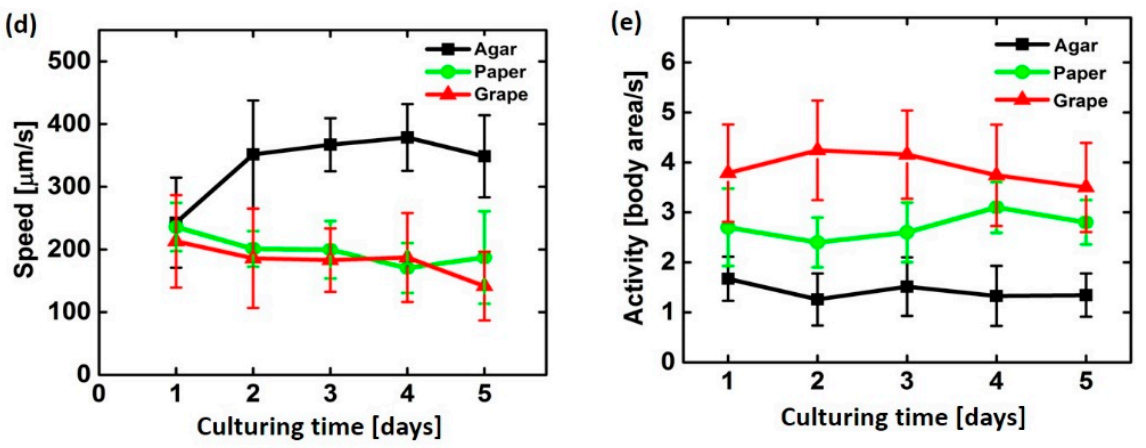

Figure 5. C. elegans' speed and activity (a) on 2-D agar plate, (b) in 3-D polycarbonate stack, and (c) in 3-D natural habitat (grape). Summary of their (d) speeds and (e) activities.

\subsection{C. elegans' Growth in 3D Culture System}

The stereological cell counting method was used to evaluate the worm proliferation in two different 9-well culturing platforms: 2D agar plate and 3D polycarbonate stack (Figure 6a) [20]. Each well was divided into four regions for better microscopic observation. First, we dropped the E. coli OP50 onto the culture platforms. Then, C. elegans at different growth states were transferred to each well and their development and fertility were observed. For better characterization, C. elegans at the L1, L2, and adult stage were counted (Figure 6b-f). Overall, the reproduction and growth rates in the $3 \mathrm{D}$ system were higher than those on the 2D plates. This is mainly because the 3D culture platform reproduces more dynamic physiological conditions of the C. elegans and provides a more effective environment for nutrient and gas supply. After culturing the worms for seven days on the $3 \mathrm{D}$ polycarbonate stack, the individual five layers of the platform were de-stacked to determine the 
distribution of the worms. Figure S2 confirms the vertical distribution of the worms, indicating that our platform forms a layered structure as a scaffold to offer the in vivo 3D environment.

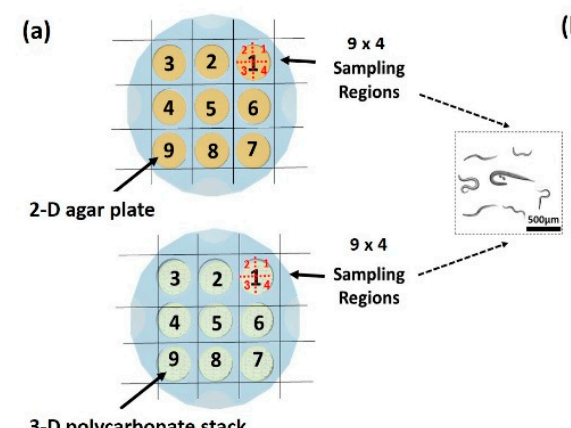

(d)

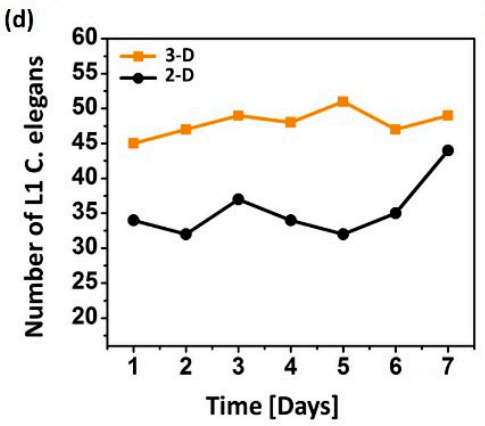

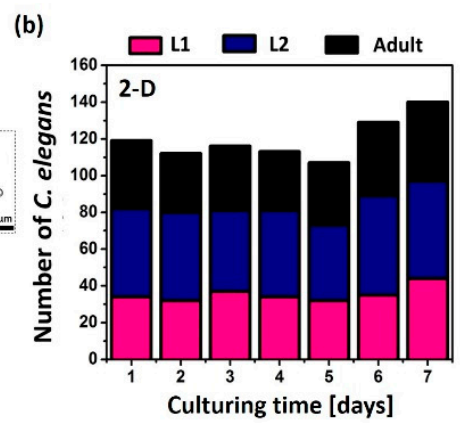

(e)

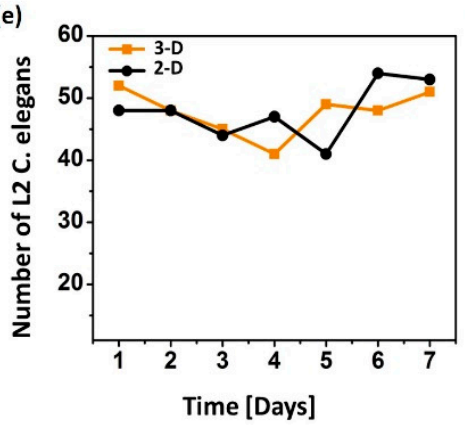

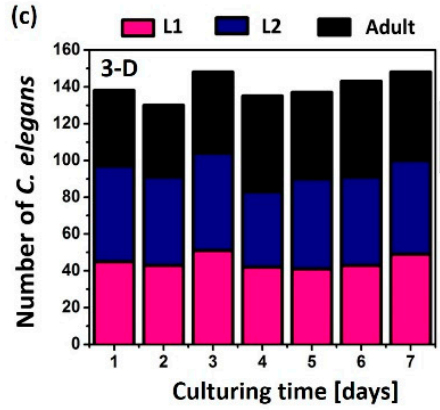

(f)

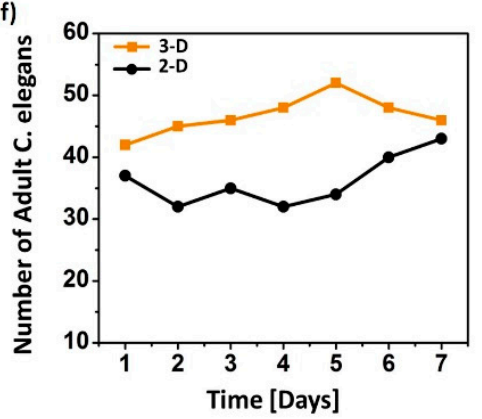

Figure 6. (a) Schematic illustration of experimental setup with two culture platforms. The number of C. elegans in L1, L2, and adult development stage (b) on the 2-D agar plate and (c) in the 3-D stack. Summary of the data with (d) L1, (e) L2, and (f) adult worms.

\section{Conclusions}

This study reports innovative paper and paper-like platforms for 3D culturing, immobilizing, and monitoring of $C$. elegans. The device was readily fabricated by stacking multiple layers of paper or paper-like polycarbonate substrates. A 96-well paper array was first developed to provide high-throughput culturing environments and in situ monitoring of the worms. Micro-pores of paper allowed C. elegans to pass through the paper structure and to be cultivated in 3D. As the worms grow and their body size become comparable to the paper pores, they can be trapped and immobilized by the paper matrix, which eases further high-throughput imaging and analysis. We also created a novel 3D cultivation platform for C. elegans by using a paper-like polycarbonate substrate. The transparency of the polycarbonate layers facilitated visualization of the worms under a microscope and allowed us to observe their behavioural dynamics in 3D. Well-defined through-holes in the 3D polycarbonate stack provided a micro-scale in vivo-like and well-controlled environment for C. elegans, improving their physical movements, growths, and proliferations. Our technique will offer powerful tools for high-throughput worm-on-a-chip manipulation and fundamental and drug discovery research in C. elegans.

\section{Experimental Sections}

\subsection{C. elegans and Culture}

Wildtype Bristol N2, age-1 mutant (TJ1052), and sir-2.1 mutant (VC199) nematode worms were obtained from the Caenorhabditis Genetics Center. All worms were maintained on nematode growth medium (NGM) agar plates containing abundant OP50 Escherichia coli cells as food at $20^{\circ} \mathrm{C}$ according to a standard protocol [21]. To produce age-synchronized worms, gravid adult hermaphrodite worms were transferred onto test systems for culturing and comparison studies. 


\subsection{Preparing E. coli OP50 Bacteria}

Escherichia coli OP50 were grown from $-80{ }^{\circ} \mathrm{C}$ glycerol stock cultures by inoculating $20 \mathrm{~mL}$ of L-broth medium with gentle shaking in air for $24 \mathrm{~h}$ at $35^{\circ} \mathrm{C}$. The L-broth media consisted of $10.0 \mathrm{~g}$ tryptone, $5.0 \mathrm{~g}$ yeast extract, and $5.0 \mathrm{~g} \mathrm{NaCl}$ per liter. The culture was then centrifuged at $5000 \mathrm{rpm}$ for $5 \mathrm{~min}$ to remove the supernatant. The bacterial cells were re-suspended in a new medium and used as a feeding inoculum for culturing systems.

\subsection{A Paper-Based 96-Well Culturing Platform}

The platform consisted of four functional layers as shown in Figure 1; a Whatman \#1 filter paper, a PMMA boundary layer, a PMMA 96-well layer, and a PMMA bottom layer. Each layer was micro-patterned by using laser micromachining (Universal Laser System, VLS3.5, Yokohama, Japan). All transparent PMMA layers were thermally bonded at $100{ }^{\circ} \mathrm{C}$ for $1 \mathrm{~h}$. The worms reproduced and were collected in each well while the fully-grown ones were trapped and immobilized by small paper pores.

\subsection{A Transparent 3D Culture System}

We chose paper-like polycarbonate as a scaffold for the model environment for C. elegans culturing. Since the polycarbonate layer is thin, transparent, mechanically strong, and soft, it is suitable for forming a 3D structure for cell culture. Polycarbonate layers have been widely used as an excellent bio-scaffold for cell distribution, adhesion, and growth, as well as allowing for fluorescence imaging without light scattering $[19,22,23]$. The PMMA layers and polycarbonate substrates were precisely cut by a laser cutting machine. All layers were aligned and assembled to define 96 wells for culturing (Figure 4).

\subsection{Stereological Cell Counting}

Counting the number of cells in a region of interest with stereology yields reliable quantitative data that can be used to determine if the number of cells differs between experimental and control groups [20]. In this work, we used a systematic random sampling (SRS) method to obtain a statistically valid sample of the region of interest (Figure 4). We divided each culturing well into four regions and recorded the quantified data.

\subsection{Fertility and Distribution Analysis}

The growth and development of multicellular organisms are critically linked to their nutritional status [24]. The four distinct larval stages (L1-L4) during C. elegans growth provide easily detectable developmental milestones. In this study, we considered the number of C. elegans at L1, L2, and adult stage as a critical indicator of culturing yield (Figure 6). After seven days of culturing, the layers were disassembled and the number of worms in 36 randomly selected regions were counted (Figure S2).

Supplementary Materials: The following are available online at http://www.mdpi.com/2072-666X/11/1/99/s1. Figure S1: (a) SEM and (b) microscopic image of polycarbonate; Figure S2: Number of C. elegans distributed in the individual layers.

Author Contributions: S.C conceived and designed the experiments; M.T. and M.M. performed the experiments; M.T. and S.C. analyzed the data; M.M. contributed reagents/materials/analysis tools; M.T. and S.C. wrote the paper; S.C. supervised the project. All authors have read and agreed to the published version of the manuscript.

Funding: This work is supported by the National Science Foundation (ECCS \#1703394), Integrated Electronics Engineering Center (IEEC), and the SUNY Binghamton Research Foundation (SE-TAE).

Conflicts of Interest: The authors declare no conflict of interest. 


\section{References}

1. Mirbagheri, M.; Adibnia, V.; Hughes, B.R.; Waldman, S.D.; Banquy, X.; Hwang, D.K. Advanced cell culture platforms: A growing quest for emulating natural issues. Mater. Horiz. 2019, 6, 45-71. [CrossRef]

2. Yeon, J.H.; Park, J. Microfluidic cell culture systems for cellular analysis. Biochip J. 2007, 1, 17-27.

3. Coluccio, M.L.; Perozziello, G.; Malara, N.; Parrotta, E.; Zhang, P.; Gentile, F.; Limongi, T.; Raj, P.M.; Cuda, G.; Candeloro, P.; et al. Microfluidic platforms for cell cultures and investigations. Microelectron. Eng. 2019, 208, 14-28. [CrossRef]

4. Dou, X.; Feng, C. Amino acids and peptide-based supramolecular hydrogels for three-dimensional cell culture. Adv. Mater. 2017, 29, 1604062.

5. Baumeister, R.; Ge, L. The worm in us-Caenorhabditis elegans as a model of human disease. Trends Biotechnol. 2002, 20, 147-148. [CrossRef]

6. Markaki, M.; Tavernarakis, N. Modeling human diseases in Caenorhabditis elegans. Biotechnol. J. 2010, 5, 1261-1276. [CrossRef] [PubMed]

7. Lee, T.Y.; Yoon, K.; Lee, J.I. NGT-3D: A simple nematode cultivation system to study Caenorhabditis elegans biology in 3D. Biol. Open 2016, 5, 529. [CrossRef]

8. Ben-Yakar, A. High-content and high-throughput in vivo drug screening platforms using microfluidics. ASSAY Drug Dev. Technol. 2019, 17, 8-13. [CrossRef]

9. Krajniak, J.; Lu, H. Long-term high-resolution imaging and culture of C. elegans in chip-gel hybrid microfluidic device for developmental studies. Lab Chip 2010, 10, 1862-1868. [CrossRef]

10. Wang, J.; Meng, J.; Ding, G.; Kang, Y.; Zhao, W. A novel microfluidic capture and monitoring method for assessing physiological damage of C. elegans under microgravity. Electrophoresis 2019, 40, 922-929. [CrossRef]

11. Bakhtina, N.A.; Korvink, J.G. Microfluidic laboratories for C. elegans enhance fundamental studies in biology. RSC Adv. 2014, 4, 4691-4709. [CrossRef]

12. Gilleland, C.L.; Rohde, C.B.; Zeng, F.; Yanik, M.F. Microfluidic immobilization of physiologically active Caenorhabditis elegans. Nat. Protoc. 2010, 5, 1888-1902. [CrossRef] [PubMed]

13. Deiss, F.; Mazzeo, A.; Hong, E.; Ingber, D.E.; Derda, R.; Whitesides, G.M. Platform for high-throughput testing of the effect of soluble compounds on 3D cell culture. Anal. Chem. 2013, 85, 8085-8094. [CrossRef] [PubMed]

14. Lewis, G.G.; DiTucci, M.J.; Baker, M.S.; Phillips, S.T. High throughput method for prototyping three-dimensional, paper-based microfluidic devices. Lab Chip 2012, 12, 2630-2633. [CrossRef] [PubMed]

15. Tahernia, M.; Mohammadifar, M.; Hassett, D.J.; Choi, S. A fully disposable 64-well papertronic sensing array for screening electroactive microorganisms. Nano Energy 2019, 65, 104026. [CrossRef]

16. Mosadegh, B.; Dabiri, B.E.; Lockett, M.R.; Derda, R.; Campbell, P.; Parker, K.K.; Whitesides, G.M. Three-dimensional paper-based model for cardiac ischemia. Adv. Healthc. Mater. 2014, 3, 1036-1043. [CrossRef]

17. Liu, H.; Li, X.; Crooks, R.M. Paper-based SlipPAD for high-throughput chemical sensing. Anal. Chem. 2013, 85, 4263-4267. [CrossRef]

18. Dong, X.; Song, P.; Liu, X. An automated microfluidic system for morphological measurement and size-based sorting of C. elegans. IEEE Trans. Nanobioscience 2019, 18, 373-380. [CrossRef]

19. Chen, Q.; He, Z.; Liu, W.; Lin, X.; Wu, J.; Li, H.; Lin, J. Engineering Cell-Compatible Paper Chips for Cell Culturing, Drug Screening, and Mass Spectrometric Sensing. Adv. Healthc. Mater. 2015, 28, 2291-2296. [CrossRef]

20. Stehman, S.V. Comparison of systematic and random sampling for estimating the accuracy of maps generated from remotely sensed data. Photogramm. Eng. Remote Sens. 1992, 58, 1343-1350.

21. Chaudhuri, J.; Parihar, M.; Pires-daSilva, A. An introduction to Worm Lab: From culturing worms to mutagenesis. J. Vis. Exp. 2011, 47, e2293. [CrossRef] [PubMed]

22. Hutak, C.M.; Kavanagh, M.E.; Reddy, I.K. Comparative Development of SIRC Rabbit Corneal Cells Grown on Polycarbonate- and Polyester-Based Filters. Skin Pharmacol. Appl. Skin Physiol. 2002, 15, 133-138. [CrossRef] [PubMed] 
23. Chang, C.; Cheng, Y.; Tu, M.; Chen, Y.; Peng, C.; Liao, W.; Tung, Y. A polydimethylsiloxane-polycarbonate hybrid microfluidic device capable of generating perpendicular chemical and oxygen gradients for cell culture studies. Lab Chip 2014, 14, 3762-3772. [CrossRef] [PubMed]

24. McDiarmid, T.A.; Yu, A.J.; Rankin, C.H. Beyond the response-High throughput behavioral analyses to link genome to phenome in Caenorhabditis elegans. Genes Brain Behav. 2018, 17, e12437. [CrossRef] [PubMed] 